\title{
DNA methylation and demethylation underlie the sex difference in estrogen receptor alpha in the arcuate nucleus
}

\author{
Laura R. Cortes ${ }^{1}$, Carla D. Cisternas ${ }^{2}$, lagn N.K.V. Cabahugํ, Damian Mason", Emma K. \\ Ramlall ${ }^{1}$, Alexandra Castillo-Ruiz ${ }^{1}$, and Nancy G. Forger ${ }^{1}$
}

\begin{abstract}
${ }^{1}$ Neuroscience Institute, Georgia State University, Atlanta, GA, USA
${ }^{2}$ Instituto de Investigación Médica Mercedes y Martín Ferrreyra INIMEC-CONICET-UNC, Córdoba Argentina
\end{abstract}

Short title: DNA methylation and de-methylation regulate estrogen receptor alpha

\section{Corresponding Author:}

Nancy G Forger, PhD

Neuroscience Institute

Georgia State University

100 Piedmont Ave SE

Atlanta, GA, 30303, USA

Phone: 404-413-5888

Email: nforger@gsu.edu

Number of Tables: 0

Number of Figures: 6

Keywords: DNMT, TET, ventromedial hypothalamus, Esr1, epigenetics 


\begin{abstract}
Introduction: Neurons expressing estrogen receptor (ER) a in the arcuate nucleus (ARC) of the hypothalamus sex-specifically control energy homeostasis and bone density. Females have more of these neurons than do males, but how this sex difference develops is unknown. Objective: We tested the hypothesis that DNA methylation and/or demethylation control the development of a sex difference in ERa in the ARC. Methods: ERa immunoreactive neurons were quantified at birth and at weaning in male, female and testosterone-treated female mice that received neonatal, intracerebroventricular injections of vehicle or zebularine, a DNA methyltransferase inhibitor. Methylation status of Esr1 was determined in the ARC and ventromedial hypothalamus (VMH) using bisulfite conversion of DNA followed by pyrosequencing. Small interfering RNAs against ten-eleven translocases were used to examine effects of demethylation on ERa cell number. Results: A sex difference in ERa cell number in the ARC, favoring females, developed between birth and weaning and was due to programming effects of testosterone. Zebularine treatment eliminated the sex difference in ERa in the ARC at weaning by decreasing ERa in females to male-like levels. Previously, the same treatment increased ERa in males in the $\mathrm{VMH}$. A promoter region of Esr1 exhibited sex differences in opposite directions in percent of total methylation in the ARC (females > males) and VMH (males > females). Moreover, neonatal inhibition of demethylation increased ERa in the ARC of males. Conclusion: DNA methylation and demethylation regulate ERa cell number in the ARC, and methyl marks may paradoxically activate Esr1 in this region.
\end{abstract}




\section{Introduction}

A transient exposure to testosterone during perinatal life underlies the emergence of many sex differences in the mammalian brain [1,2]. For example, male mice have more neurons expressing calbindin and vasopressin in several forebrain regions [3,4], while females have more neurons expressing estrogen receptor (ER) $\alpha[5,6]$. In some cases, sex differences in developmental cell death is prevented, e.g., $[7,8]$. In the latter case, sexual differentiation is often a process of establishing stable, sex-specific patterns of gene expression in neurons, i.e., the differentiation of neurochemical phenotype. Although sex differences in neurochemical phenotype are common, the underlying molecular processes are largely unknown.

The arcuate nucleus (ARC) is located in the mediobasal hypothalamus and exhibits more ER $\alpha$ expressing neurons in females than in males, at least in rats [6]. ER $\alpha$ neurons in the ARC play important roles in the control of feeding behavior, energy homeostasis, and the regulation of bone density [9-11], but what causes females to have more of these neurons is unknown.

Epigenetic modifications, such as DNA methylation, play prominent roles in the differentiation of cell type throughout the body and may underlie sexual differentiation of ER $\alpha$ neurons [12-16]. For example, we recently found that inhibiting DNA methylation in newborn mice reduces or eliminates the usual female bias in the number of neurons expressing ER $\alpha$ in the preoptic area and ventrolateral area of the ventromedial nucleus of the hypothalamus $(\mathrm{VMHvl})$, which lies adjacent to the ARC $[14,16]$.

DNA methylation is catalyzed by DNA methyltransferases (DNMTs) and usually occurs at the 5th carbon position of cytosine residues followed by a guanine (CpG sites) [17]. DNA methyl marks (5methylcytosine; $5 \mathrm{mC}$ ) can be quite long-lasting [18], but they can also be removed through a series of oxidative steps catalyzed by ten-eleven translocation enzymes (TETs). In the first step, $5 \mathrm{mC}$ is converted to 5 -hydroxymethylcytosine $(5 \mathrm{hmC})$, which is both an intermediary in demethylation and an independent epigenetic mark in its own right [19]. Although a growing number of exceptions are reported [20-25], $5 \mathrm{mC}$ is typically associated with gene repression, whereas $5 \mathrm{hmC}$ is most often associated with gene expression [22,26-28]. Thus, the emergence of a particular cell phenotype in part depends on the balance between methylation (controlled by DNMTs) and demethylation (controlled by TETs) at specific genes.

The methylation landscape of the brain changes dynamically during the first few weeks (rodents) or years (humans) of life [29]. We recently found that the expression and enzyme activity of DNMTs and TETs peak in the mouse hypothalamus during the first postnatal week [30], which coincides with the 
critical period for sexual differentiation of the brain and behavior. Furthermore, we found sex differences in Tet gene expression in the neonatal hypothalamus, with greater expression of Tet2 and Tet3 in males [30]. Here, we manipulated DNMTs and TETs neonatally to test the hypothesis that DNA methylation and/or demethylation underlie sexual differentiation of ER $\alpha$ in the ARC. We also examined DNA methylation of Esr1 promoter regions in both the ARC and neighboring VMHvl.

\section{Materials and Methods}

Animals

C57BL6/J mice were bred in our vivarium and checked daily for pups. Mice were housed in cages with corn cob bedding in a room maintained at $22^{\circ} \mathrm{C}$ with food and water available ad libitum. Animal procedures were performed in accordance with the National Institutes of Health animal welfare guidelines and were approved by the Georgia State University Institutional Animal Care and Use Committee.

Neonatal testosterone treatment and DNA methyltransferase inhibition

The brains used in this portion of the study were the same as those used in a prior study reporting on the effects of a neonatal inhibition of DNA methylation on ER $\alpha$ in the POA and VMHvl [16]. Male and female controls received a subcutaneous injection of $25 \mu \mathrm{L}$ of peanut oil on the day of birth and following day, while androgenized females received testosterone propionate (100 $\mu \mathrm{g}$ in $25 \mu \mathrm{L}$ peanut oil; Millipore Sigma, St Louis, MO). Half of the animals in each of the three hormonal groups also received intracerebroventricular (ICV) injections of either vehicle or a DNA methyltransferase inhibitor, zebularine, on the day of birth (postnatal day (P) 0) and P1. Vehicle $(500 \mathrm{~nL}$ per hemisphere; $10 \%$ dimethyl sulfoxide in 90\% saline) and zebularine (300 ng in $500 \mathrm{~nL}$ vehicle; Millipore Sigma) were injected into the lateral ventricles of cryoanesthetized pups, as described previously [16]. All animals were tattooed for identification and were sacrificed prior to puberty at P25. This age was chosen because it allowed us to examine relatively long-lasting effects of the neonatal inhibition of DNA methylation in the absence of confounding effects of post-pubertal gonadal hormones.

\section{Neonatal Tet inhibition}

Tet mRNA expression in the hypothalamus is elevated during the first postnatal week, and sex differences in Tet2 and Tet3 expression (male > female) are observed during this time [30]. To determine the role of demethylation in the development of the sex difference in ERa expression in the 
ARC and $\mathrm{VMHvl}$, we used small interfering RNAs (siRNAs) to reduce Tet expression in males to femalelike levels. ICV injections were performed on cryoanesthetized pups as described above. On P5, females received a control injection of non-targeting RNAs in delivery media while males received either the control or a cocktail of SMARTPool siRNAs targeted against Tet2 and Tet3 (400-500 pmol; Accell, Horizon Discovery, Cambridge, UK). Each SMARTPool contains a mixture of four siRNAs targeting the same mRNA. Previous studies using Accell siRNAs report a neuron-selective knock-down that is maximal 2-4 days after a single ICV injection [31,32]. Thus, a subset of brains was collected two days after injection (P7), and punches were taken of the anterior and posterior hypothalamus to confirm gene knock-down by RT-qPCR, as described in [30]. The remaining brains were harvested at P25, immunohistochemically stained for ERa, and the ARC and VMHvl were analyzed as described below.

Immunohistochemistry and quantification of ERa

Brains were collected and fixed in $5 \%$ acrolein in $0.1 \mathrm{M}$ phosphate buffer for $24 \mathrm{~h}$, then transferred to $30 \%$ sucrose in $0.1 \mathrm{M}$ phosphate buffer for several days. Brains were frozen-sectioned into four series (zebularine-treated animals) or two series (siRNA-treated animals) of $30 \mu \mathrm{m}$ thickness and stored in cryoprotectant (30\% sucrose, 30\% ethylene glycol, and 1\% polyvinylpyrrolidone in $0.1 \mathrm{M}$ phosphate buffer) until immunohistochemical staining. One series was stained for ERa (rabbit anti-ERa, 1:20,000; EMD Millipore, Billerica, MA) as described previously [16]. A separate cohort of P1 untreated tissue was also immunostained for ERa to determine if sex differences were present at this age. Images through the ARC were captured using Stereo Investigator software (MBF Bioscience, Williston, VT) and ERa labeling was analyzed using ImageJ (Version 1.52/1.53; National Institutes of Health, Bethesda, MD). The boundaries of the ARC were traced and the number of pixels above threshold was quantified per $\mu \mathrm{m}^{2}$. Pixels above background were automatically determined using the Shanbhag algorithm, and the four sections through the ARC with the greatest labeling were summed for each animal. To validate this method, we also manually counted ERa cells in a subset of male and female animals using Stereo Investigator and confirmed a very similar pattern of results.

\section{Quantification of cell death}

To determine whether zebularine treatment altered developmental cell death, brains from a separate cohort of vehicle- and zebularine-treated pups were collected 6 hours after the last injection on P1 and immunolabeled for activated caspase-3 (AC3) as previously described [33]. We drew contours around 
the ARC and counted the number of AC3-positive cells bilaterally. Cell death density was obtained by dividing the number of AC3-positive cells by the area sampled and is expressed as AC3 cells per $\mathrm{mm}^{2}$. Stereological Cell Counts

A separate series of brain sections from vehicle- and zebularine-treated females was stained with thionin to determine whether zebularine altered the total number of cells in the ARC at weaning. Unbiased stereology using the optical fractionator probe was performed using Stereo Investigator software (MBF Biosciences). Contours were drawn around the region of interest at low power, and counts of cells exhibiting a neuronal morphology were performed using the 100x oil objective within a $324 \mu \mathrm{m}^{2}$ counting frame and using a 4,900 $\mu^{2}$ sampling grid. The Gundersen coefficient of error for counts was between $5-7 \%$ [34].

\section{Bisulfite mapping of Esr1 in the ARC and VMHvl}

A separate cohort of male $(n=84)$ and female $(n=84)$ pups received ICV injections of vehicle or zebularine on P0 and P1. Forty-eight vehicle-treated pups ( 24 of each sex) were sacrificed $\sim 4 \mathrm{~h}$ after ICV injection on P1, and all remaining animals were collected on P25. Brains were removed, flash-frozen in 2-methylbutane cooled to $-20^{\circ} \mathrm{C}$, and cut on a cryostat to the level of the ARC and VMHvl. Punches were taken from the $\mathrm{ARC}$ and $\mathrm{VMHvl}$ and kept at $-80^{\circ} \mathrm{C}$ until processing. Because a punch of the ARC damages the adjacent $\mathrm{VMHvl}$, and vice versa, separate animals were used for each brain region. Punches from three animals of the same sex were pooled per sample. Bisulfite conversion followed by pyrosequencing was performed by EpigenDx (Hopkinton, MA) to obtain single-nucleotide resolution of modified cytosines in Esr1. Specifically, we assessed the modification of cytosines in $16 \mathrm{CpG}$ sites in the untranslated regions of Exons $A$ and $C$. Because bisulfite sequencing cannot distinguish between $5 \mathrm{mC}$ and $5 \mathrm{hmC}$, we refer to the results of this analysis as "total methylation" (as in [22,35]). Methylation of these regions was previously shown to associate with changes in Esr1 gene expression in the mouse brain during development or after bisphenol A exposure [36,37].

\section{$\underline{\text { Statistics }}$}

Independent, two-tailed t-tests were used to compare ER $\alpha$ labeling in the ARC of untreated males and females on P1. Two-way ANOVA (group-by-treatment) was used to compare ER $\alpha$ labeling at P25 in males, females, and testosterone-treated females that received either vehicle or zebularine neonatally. Results of pyrosequencing were analyzed using a two-way repeated measures ANOVA for P1 samples, with sex as the between-subjects factor and CpG site as the repeated measure. Pyrosequencing at P25 
was analyzed with a three-way repeated measure ANOVA with sex and zebularine treatment as between-subjects factors and CPG site as the repeated measure. A significant main effect of sex was followed by post-hoc tests of individual CpG sites. Developmental changes in mean methylation across CpG sites were analyzed with two-way ANOVAs (age-by-sex). Effect of Tet siRNA treatment on ER $\alpha$ expression was analyzed by one-way ANOVA. Fisher's LSD's post-hoc or Tukey's tests were used where appropriate and all analyses were performed using Prism Version 9 (GraphPad Software, San Diego, CA).

\section{Results}

\section{A sex difference in ERa cell number in the ARC is programmed by neonatal testosterone and eliminated by an inhibition of DNA methylation}

We observed prominent ER $\alpha$ labeling in the ARC of untreated animals that appeared confined to cell nuclei (Figure 1). There was no sex difference at P1 (Fig. 1a,c), but females had more ER $\alpha$ labeling than males at weaning (P25; $\mathrm{t}_{16}=3.64, P=0.002$; Fig. $\left.1 \mathrm{~b}, \mathrm{~d}\right)$. Thus, the sex difference in ER $\alpha$ in the ARC previously reported in adult rats [6] is present prior to puberty in mice. To examine hormonal control of the sex difference, we compared ERa in the ARC at P25 in the males, females, and females treated neonatally with testosterone from our previous study [16]. As expected, control females had greater ER $\alpha$ labeling than did control males $(P<0.0001$ for Vehicle Female vs Vehicle Male; Fig. 2). Neonatal treatment of females with testosterone markedly reduced ER $\alpha$ labeling at P25 $(P<0.0001$ for Vehicle Female vs Vehicle Female $+T$ ), indicating that the sex difference is due to programming effects of testosterone. Our testosterone treatment, in fact, hyper-masculinized females relative to vehicletreated males $(P<0.01)$.

To probe the role of DNA methylation, we compared ERa labeling in the ARC in animals treated neonatally with zebularine or vehicle. There was no main effect of neonatal zebularine treatment, but a highly significant interaction between group (Male, Female, and Female $+\mathrm{T}$ ) and treatment (Vehicle vs. Zebularine; $F_{2,54}=15.12, P<0.0001 ; F i g$. 2). Neonatal zebularine treatment decreased ER $\alpha$ labeling in control females $(P<0.0005)$ to male-like levels, while having no effect on males. Zebularine also slightly increasing labeling in testosterone-treated females $(P=0.035)$. As a result, group differences in $\mathrm{ER} \alpha$ labeling in the ARC were eliminated among the zebularine-treated animals (Fig. 2). The same pattern of results was obtained using direct cell counts of ERa, rather than automated analyses of labeling (not shown). The effects of zebularine were independent of a change in developmental cell death in both sexes at P1 (Fig. 3a), or total cell number in females at P25 (Fig. 3b). Thus, neonatal inhibition of DNA 
methylation reduced the proportion of neurons that express ER $\alpha$ in females, rather than the number of surviving neurons.

The results of this experiment were somewhat surprising because neonatal zebularine treatment eliminates the sex difference in ERa in the VMHvl by increasing expression specifically in males and testosterone-treated females, with no effect in females $[14,16]$. That is, a neonatal inhibition of DNA methylation leads to high, female-like ERa expression across groups in the VMHvl [16], but uniformly low, male-like ERa expression across groups in the neighboring ARC of the same animals. This suggested that DNA methylation regulates ERa expression very differently in these neighboring brain regions.

\section{Opposite effects of sex on DNA methylation of Esr1 in the ARC and VMHvl}

We used bisulfite conversion of DNA followed by pyrosequencing to compare effects of age, sex, and zebularine treatment on total methylation $(5 \mathrm{mC}+5 \mathrm{hmC})$ of the ERa gene, Esr1, in the ARC and VMHvl. We focused on $16 \mathrm{CpG}$ sites in two promoter regions shown to regulate expression of Esr1 in the mouse brain: CpGs 1-11 in untranslated Exon $A$ and $C p G s$ 40-44 in untranslated Exon C [36,37].

The levels of total methylation of Esr1 in the ARC and VMHvl observed at P1 were similar to those reported by Westberry et al. [36] in the neonatal mouse cortex using the same technique. In Exon A, repeated measures ANOVA indicated no overall sex difference in total methylation across the $11 \mathrm{CpG}$ sites in either the ARC or VMHvl on P1 (Fig. 4a). However, sex differences in both brain regions emerged at P25, and in opposite directions: total methylation levels across the $11 \mathrm{CpG}$ sites was significantly higher in females than males in the ARC (main effect of sex, $F_{1,16}=8.63, P<0.01$ ), and higher in males than in females in the VMHvl $\left(F_{1,16}=7.69, P<0.015\right.$; Fig. 4a). Analyses of individual $\mathrm{CpG}$ sites showed that the pattern ( $F>M$ in the $A R C$, and $M>F$ in the $V M H v l$ ) was consistent across most $C p G$ sites (Fig. 4b). However, the difference reached significance only for CpG 10 in the ARC, and CpGs 6, 9, 10, and 11 in the VMHvl. The effects of sex were also specific to Exon $\mathrm{A}$, as we found no sex differences in Exon $\mathrm{C}$ at P1 or at P25 in either the ARC or VMH (all P-values $>0.05$; Suppl. Fig. 1).

The sex differences that emerged at P25 were superimposed upon large increases in average total methylation of $\mathrm{CpG}$ sites of the Exon A promoter region in both sexes and regions between P1 and P25 (main effect of age; $F_{1,24}>200, P<0.0001$ in each region; Fig. 4a), consistent with our previous observation of an overall increase in global $5 \mathrm{mC}$ between birth and P25 in the mouse hypothalamus [30]. In addition, we found an interaction between age and sex in both regions (ARC: $F_{1,24}=5.01, P=$ 0.035; VMH: $\left.F_{1,24}=7.59, P=0.011\right)$, due to the fact that females gained more methylation with age than males in the ARC, and males gained more methylation with age than females in the VMHvl. There was a 
smaller increase in total methylation with age in Exon C when collapsing across sex (main effect of age; ARC: $F_{1,24}=5.41, P=0.029 ; V_{M H v l: ~} F_{1,24}=6.52, P=0.017$; Suppl. Fig. 1). Paradoxically, we also found that neonatal zebularine treatment increased total methylation in Exon $A$ at $P 25$ in the $A R C\left(F_{1,16}=\right.$ 10.43, $P<0.01$; Suppl. Fig. 2), with no sex-by-treatment interaction. Post hoc tests indicate that the effect was significant for CpG sites 6 and 9 (not shown). There was no effect of neonatal zebularine treatment on total methylation in the VMHvl.

\section{Down-regulation of Tet expression increases ERa in the ARC of males}

The brain exhibits dynamic changes in DNA methylation during development [29,38,39], indicating an active methylation/demethylation cycle. TET enzymes control the turnover of DNA methyl marks, and mRNA expression of Tet2 and Tet3 is higher in neonatal males than in females in the POA and mediobasal hypothalamus [30]. This suggests that de-methylation may play a role in the development of sex differences. To test this, we administered siRNAs targeting Tet2 and Tet3 to newborn males, while control males and females received injections of non-targeting RNAs. Tet2/Tet3 expression was significantly reduced in punches of the anterior and posterior hypothalamus $48 \mathrm{~h}$ after siRNA injection (main effect of siRNA, Tet2: $F_{1,10}=6.72, P=0.027$; Tet3: $F_{1,10}=5.21, P<0.05$; Suppl. Fig. 3). The reduction was fairly subtle ( $15-30 \%$ ), but comparable to the magnitude of sex differences in Tet expression seen previously [30]. To determine whether this partial knock-down was functionally significant, ER $\alpha$ labeling was examined at P25.

In the ARC, we found a significant difference in ER $\alpha$ labeling across the three groups $\left(F_{2,20}=4.66, P<\right.$ 0.025 ) and, as expected, females had greater ER $\alpha$ labeling than control males $(P<0.04)$. In males with Tet2/Tet3 knock-down, ER $\alpha$ labeling was significantly increased relative to control males $(P<0.015)$, to a level very similar to that of females ( $P=0.97 ;$ Fig. 5$)$. In contrast, siRNAs against Tet 2 and Tet3 had no effect on ER $\alpha$ labeling in the VMHvl, although we did find the expected sex difference, with greater ER $\alpha$ labeling in females $\left(F_{2,21}=14.94, P<0.0001\right)$.

\section{Discussion}

ER $\alpha$ neurons are highly enriched in the ARC and $\mathrm{VMHvl}$, and play important roles in estradiolmediated effects on energy homeostasis. A knockdown of Esr1 in the ARC increases food intake and bone density in female mice $[10,11]$, whereas blocking ERa signaling in the $\mathrm{VMHvl}$ reduces energy expenditure and thermogenesis $[11,40,41]$. In both regions, effects are sex-specific, with little or no effect of Esr1 knockdown on energy homeostasis in males [9-11,42]. Females have more ERa neurons 
in both the ARC and $\mathrm{VMHVl}$, and a similar female bias in ER $\alpha$ cell number has been reported in a variety of brain regions and vertebrate species [43-47]. In several cases, the sex difference requires differential exposure to testosterone during perinatal life [48-50], but the mechanism(s) by which testosterone programs sex differences in ER $\alpha$ expression, or other sex differences in neurochemistry for that matter, remains largely unknown.

We previously reported that sex differences in ER $\alpha$ in the POA and VMHvl of mice develop postnatally and are epigenetically regulated [14,30]. Both sexes express high levels of ER $\alpha$ at birth, and ER $\alpha$ cell number decreases in males over the next few weeks. The decrease can be prevented by treating neonatal males with an inhibitor of DNA methyltransferases, without an effect on cell death or total cell number [14]. We showed here that a sex difference favoring females also develops postnatally in the ARC and is abolished by inhibiting DNMTs during the first two days of life. However, in contrast to the POA and VMHvl, DNMT inhibition eliminated the sex difference in the ARC by decreasing (i.e., masculinizing) ER $\alpha$ expression in females.

We also found sex differences in total methylation of a promoter region of Esr1 that emerged at weaning. Across $11 \mathrm{CpG}$ sites of Exon A, males had a higher percent of total methylation than females in the $\mathrm{VMHvl}$ at $\mathrm{P} 25$. This is consistent with the hypothesis that $5 \mathrm{mC}$ marks contribute to the lower $\mathrm{ER} \alpha$ labeling in the $\mathrm{VMHvl}$ of males. In the ARC, however, females had a higher percent of total methylation across Exon A, despite their higher levels of ER $\alpha$. In addition, neonatal inhibition of DNMTs reduced ER $\alpha$ expression in the ARC of females, and neonatal knock-down of Tet2/Tet3 expression in males, which is expected to increase $5 \mathrm{mC}$, increased ER $\alpha$ cell number at weaning. Taken together, these observations suggest that $5 \mathrm{mC}$ marks across the $\mathrm{CpG}$ sites of Exon A are associated with Esr1 gene activation in the ARC. Although contrary to the canonical association of DNA methylation with gene repression, there are a growing number of cases where DNA methylation of specific gene regions promotes transcription [2025].

One limitation of our findings is that pyrosequencing of sodium bisulfite-treated DNA does not distinguish $5 \mathrm{mC}$ marks from $5 \mathrm{hmC}$. The concern in this case is lessened by the fact that $5 \mathrm{hmC}$ in neurons primarily accumulates in gene bodies and is depleted in promoter regions [29,35]. In addition, 5mC is 45 times more abundant than $5 \mathrm{hmC}$ in $\mathrm{CpG}$ dinucleotides [51]. Because we examined promoter regions and modifications in the CpG context, most of the total methylation we detected was presumably $5 \mathrm{mC}$. Nonetheless, one would ideally like to sequence using techniques that distinguish $5 \mathrm{mC}$ from $5 \mathrm{hmC}$ [52], although the amount of starting material required is a serious impediment for this type of analysis. 
The sex differences we detected in total methylation of Esr1 were small (on the order of $2 \%$ in absolute terms and $20 \%$ relative differences between sexes across the $\mathrm{CpG}$ sites of Exon A), but similar in magnitude to what was previously reported for steroid receptors in the rat POA and mediobasal hypothalamus [39]. In our study and related previous studies [36,39,53], pyrosequencing of Esr1 was examined from brain punches, which contain many cells types. ER $\alpha$ neurons comprise only a minority of all cells, even in regions such as the POA, VHM and ARC, where they are relatively abundant. Any "signal" (i.e., a sex difference in methylation of Esr1) must therefore be detected over quite a bit of noise. In addition, any measure of DNA methylation provides evidence that is correlative in nature. To test whether any given methyl mark causes a sex difference in ER $\alpha$ cell number would require specifically manipulating that mark, and while this is now theoretically possible $[54,55]$, it may be some time before this can be achieved site-specifically, in vivo, in a newborn mouse brain.

In addition to the sex differences in total methylation that emerged at P25, we found that both sexes accumulate modified cytosines in Exon A, and to a lesser degree in Exon C, of Esr1 from birth to weaning. This is consistent with previous findings of marked increases in $5 \mathrm{mC}$ and $5 \mathrm{hmC}$ throughout the genome in the mouse hypothalamus and neocortex from birth to adolescence $[29,30]$. The fact that females have persistently high levels of ER $\alpha$ in both the ARC and VMHvl despite the accumulation of methylation in a brain-relevant Esr1 promoter, suggests that there are other regulatory mechanisms that allow for gene expression across development.

We expected zebularine, which inhibits DNMTs, to decrease $5 \mathrm{mC}$ marks and, therefore, total DNA methylation of Esr1, but did not observe that in either the VMHvl or ARC. In the VMHvl, there was no effect of neonatal zebularine treatment on total methylation of Exon A or Exon C, even though this treatment previously led to a lasting increase in ER $\alpha$ in males $[14,16]$. This suggests that zebularine may act indirectly (e.g. by inhibiting an inhibitor of Esr1 in males), or that the Esr1 promoter regions we examined do not capture the direct effects of zebularine. Although Exons $A$ and $C$ are important for developmental regulation of ER $\alpha$ in the brain, other promoters regulate ER $\alpha$ in other tissues [56], and methylation in non-promoter regions can also regulate gene expression $[24,57,58]$. Even more surprising, neonatal zebularine treatment increased the percent total methylation in Exon A of Esr1 in the $A R C$ at $P 25$. If $5 \mathrm{mC}$ indeed activates Esr1 in the ARC, the increased total methylation after zebularine would be consistent with the increased ER $\alpha$ seen in testosterone-treated females that received zebularine. However, the effect of zebularine was seen across all groups, which is harder to explain. We note that in post hoc analyses, the individual CpG sites affected by zebularine did not overlap with those that showed a significant sex difference in the ARC. In the current study, for example, 
CpG-10 was significantly different by sex, but not by zebularine treatment, in both the ARC and VMHvl. Differences in Esr1 expression between peripheral tissues have been attributed to the methylation status of a single CpG site [59], and the effects of maternal behavior on glucocorticoid receptor gene expression in the brains of offspring have also been linked to a single CpG [60].

DNA demethylation and $5 \mathrm{hmC}$ marks are just beginning to be explored in the context of sexual differentiation. Given the unusually high abundance of $5 \mathrm{hmC}$ in gene bodies of neurons [61], it is likely to have an important role in shaping neural gene expression. We recently found more consistent sex differences in the expression of Tets than of Dnmts in the neonatal mouse brain [30], with higher Tet2 and Tet 3 expression in males. The fact that suppressing Tet 2 and Tet 3 expression in males increased ER $\alpha$ in the ARC to female-like levels indicates that this sex difference is functionally meaningful. Thus demethylation, or possibly stable $5 \mathrm{hmC}$ marks, likely contribute to sexual differentiation of the brain.

Overall, our data suggest that neurons with the potential to express ER $\alpha$ are normally inhibited from doing so by the presence of DNA methyl marks in the $\mathrm{VMH}$ and the absence of such marks in the ARC. Since sex differences in both regions are due to perinatal gonadal steroids, and can be quite stable [62], hormones likely program ER $\alpha$ cell number through epigenetic mechanisms. In cancer cells, total methylation levels are reduced in response to estradiol [63], and one mechanism for this hormonedependent hypomethylation is the upregulation of Tet2 expression [64]. Neonatal estradiol also dampens the catalytic efficiency of DNMTs in the POA of the rat brain [12]. Thus, there may be parallel mechanisms by which sex steroid hormones influence DNA methylation, leading to stable changes in neurochemistry in the developing brain. 


\section{Acknowledgements}

We are appreciative of Taylor Hite for technical assistance and to members of the Forger Lab for feedback on the manuscript.

\section{Statement of Ethics}

All procedures were performed in accordance with the National Institutes of Health animal welfare guidelines and were approved by the Georgia State University Institutional Animal Care and Use Committee.

\section{Conflict of Interest Statement}

There is no conflict of interest regarding this work.

\section{Funding Sources}

This work was supported by a National Science Foundation Graduate Research Fellowship awarded to LRC, and a National Institutes of Health Grant R01 MH068482 and Brains \& Behavior Seed Grant from Georgia State University awarded to NGF.

\section{Author Contributions}

LRC, CC, and NGF conceptualized the experiments. LRC, INKVC, and NGF wrote the manuscript. LRC, CC, INKVC, DM, EKR, and ACR performed data collection and/or analysis. All authors edited and approved the manuscript. 


\section{References}

1 Lenz KM, Nugent BM, McCarthy MM. Sexual differentiation of the rodent brain: dogma and beyond. Frontiers in Neuroscience. 2012;6. DOI: 10.3389/fnins.2012.00026

2 McCarthy MM, Nugent BM, Lenz KM. Neuroimmunology and neuroepigenetics in the establishment of sex differences in the brain. Nat Rev Neurosci. 2017 Aug;18(8):471-84.

3 Edelmann M, Wolfe C, Scordalakes EM, Rissman EF, Tobet S. Neuronal nitric oxide synthase and calbindin delineate sex differences in the developing hypothalamus and preoptic area.

Developmental Neurobiology. 2007;67(10):1371-81.

4 Rood BD, Stott RT, You S, Smith CJW, Woodbury ME, De Vries GJ. Site of origin of and sex differences in the vasopressin innervation of the mouse (Mus musculus) brain. J Comp Neurol. 2013 Jul;521(10):2321-58.

5 DonCarlos LL, Handa RJ. Developmental profile of estrogen receptor mRNA in the preoptic area of male and female neonatal rats. Brain Res Dev Brain Res. 1994 Jun;79(2):283-9.

6 Kühnemann S, Brown TJ, Hochberg RB, MacLusky NJ. Sex differences in the development of estrogen receptors in the rat brain. Hormones and Behavior. 1994 Dec;28(4):483-91.

7 de Vries GJ, Jardon M, Reza M, Rosen GJ, Immerman E, Forger NG. Sexual differentiation of vasopressin innervation of the brain: cell death versus phenotypic differentiation. Endocrinology. 2008 Sep;149(9):4632-7.

8 Gilmore RF, Varnum MM, Forger NG. Effects of blocking developmental cell death on sexually dimorphic calbindin cell groups in the preoptic area and bed nucleus of the stria terminalis. Biol Sex Differ. 2012 Feb;3:5.

9 Frank A, Brown LM, Clegg DJ. The role of hypothalamic estrogen receptors in metabolic regulation. Frontiers in Neuroendocrinology. 2014 Oct;35(4):550-7.

10 Herber CB, Krause WC, Wang L, Bayrer JR, Li A, Schmitz M, et al. Estrogen signaling in arcuate Kiss1 neurons suppresses a sex-dependent female circuit promoting dense strong bones. Nature Communications. 2019 Jan;10(1):163.

11 Xu Y, Nedungadi TP, Zhu L, Sobhani N, Irani BG, Davis KE, et al. Distinct hypothalamic neurons mediate estrogenic effects on energy homeostasis and reproduction. Cell Metab. 2011 Oct;14(4):453-65.

12 Nugent BM, Wright CL, Shetty AC, Hodes GE, Lenz KM, Mahurkar A, et al. Brain feminization requires active repression of masculinization via DNA methylation. Nature Neuroscience. 2015 May;18(5):690-7.

13 Forger NG. Epigenetic mechanisms in sexual differentiation of the brain and behaviour. Philos Trans R Soc Lond B Biol Sci. 2016 Feb;371(1688). DOI: 10.1098/rstb.2015.0114 
14 Mosley M, Weathington J, Cortes LR, Bruggeman E, Castillo-Ruiz A, Xue B, et al. Neonatal inhibition of DNA methylation alters cell phenotype in sexually dimorphic regions of the mouse brain. Endocrinology. 2017 01;158(6):1838-48.

15 Manoli DS, Tollkuhn J. Gene regulatory mechanisms underlying sex differences in brain development and psychiatric disease. Ann N Y Acad Sci. 2018 May;1420(1):26-45.

16 Cisternas CD, Cortes LR, Golynker I, Castillo-Ruiz A, Forger NG. Neonatal inhibition of DNA methylation disrupts testosterone-dependent masculinization of neurochemical phenotype. Endocrinology. 2020 Jan;161(1). DOI: 10.1210/endocr/bqz022

17 Adams RL. DNA methylation. The effect of minor bases on DNA-protein interactions. Biochem J. 1990 Jan;265(2):309-20.

$18 \operatorname{Kim~M,~Costello~J.~DNA~methylation:~an~epigenetic~mark~of~cellular~memory.~Experimental~\& ~}$ Molecular Medicine. 2017 Apr;49(4):e322-e322.

19 Bachman M, Uribe-Lewis S, Yang X, Williams M, Murrell A, Balasubramanian S. 5Hydroxymethylcytosine is a predominantly stable DNA modification. Nature Chemistry. 2014 Dec;6(12):1049-55.

20 Rishi V, Bhattacharya P, Chatterjee R, Rozenberg J, Zhao J, Glass K, et al. CpG methylation of halfCRE sequences creates C/EBPalpha binding sites that activate some tissue-specific genes. Proc Natl Acad Sci U S A. 2010 Nov;107(47):20311-6.

21 Wan J, Oliver VF, Wang G, Zhu H, Zack DJ, Merbs SL, et al. Characterization of tissue-specific differential DNA methylation suggests distinct modes of positive and negative gene expression regulation. BMC Genomics. 2015 Feb;16(1). DOI: 10.1186/s12864-015-1271-4

22 Irwin RE, Thakur A, O' Neill KM, Walsh CP. 5-Hydroxymethylation marks a class of neuronal gene regulated by intragenic methylcytosine levels. Genomics. 2014 Nov;104(5):383-92.

23 Semaan SJ, Dhamija S, Kim J, Ku EC, Kauffman AS. Assessment of Epigenetic Contributions to Sexually-Dimorphic Kiss1 Expression in the Anteroventral Periventricular Nucleus of Mice. Endocrinology. 2012 Apr;153(4):1875-86.

24 Yang X, Han H, De Carvalho DD, Lay FD, Jones PA, Liang G. Gene body methylation can alter gene expression and is a therapeutic target in cancer. Cancer Cell. 2014 Oct;26(4):577-90.

25 Ball MP, Li JB, Gao Y, Lee J-H, LeProust EM, Park I-H, et al. Targeted and genome-scale strategies reveal gene-body methylation signatures in human cells. Nat Biotechnol. 2009 Apr;27(4):361-8.

26 Bird A. DNA methylation patterns and epigenetic memory. Genes Dev. 2002 Jan;16(1):6-21.

27 Lister R, Pelizzola M, Dowen RH, Hawkins RD, Hon G, Tonti-Filippini J, et al. Human DNA methylomes at base resolution show widespread epigenomic differences. Nature. 2009 Nov;462(7271):315-22.

28 Gross JA, Pacis A, Chen GG, Barreiro LB, Ernst C, Turecki G. Characterizing 5-hydroxymethylcytosine in human prefrontal cortex at single base resolution. BMC Genomics. 2015 Sep;16(1):672. 
29 Lister R, Mukamel EA, Nery JR, Urich M, Puddifoot CA, Johnson ND, et al. Global epigenomic reconfiguration during mammalian brain development. Science. 2013 Aug;341(6146):1237905.

30 Cisternas CD, Cortes LR, Bruggeman EC, Yao B, Forger NG. Developmental changes and sex differences in DNA methylation and demethylation in hypothalamic regions of the mouse brain. Epigenetics. 2020 Feb;15(1-2):72-84.

31 Hazzan T, Guhl S, Artuc M, Franke K, Worm M, Zuberbier T, et al. An efficient method for gene knock-down by RNA interference in human skin mast cells. Experimental Dermatology. 2017;26(11):1136-9.

32 Nakajima H, Kubo T, Semi Y, Itakura M, Kuwamura M, Izawa T, et al. A rapid, targeted, neuronselective, in vivo knockdown following a single intracerebroventricular injection of a novel chemically modified siRNA in the adult rat brain. J Biotechnol. 2012 Jan;157(2):326-33.

33 Ahern TH, Krug S, Carr AV, Murray EK, Fitzpatrick E, Bengston L, et al. Cell death atlas of the postnatal mouse ventral forebrain and hypothalamus: effects of age and sex. J Comp Neurol. 2013 Aug;521(11):2551-69.

34 Gundersen HJ, Jensen EB, Kiêu K, Nielsen J null. The efficiency of systematic sampling in stereology-reconsidered. J Microsc. 1999 Mar;193(Pt 3):199-211.

35 He Y, Jang HS, Xing X, Li D, Vasek MJ, Dougherty JD, et al. DeepH\&amp;M: Estimating single-CpG hydroxymethylation and methylation levels from enrichment and restriction enzyme sequencing methods. Science Advances. 2020 Jul;6(27):eaba0521.

36 Westberry JM, Trout AL, Wilson ME. Epigenetic regulation of estrogen receptor alpha gene expression in the mouse cortex during early postnatal development. Endocrinology. 2010 Feb;151(2):731-40.

37 Kundakovic M, Gudsnuk K, Franks B, Madrid J, Miller RL, Perera FP, et al. Sex-specific epigenetic disruption and behavioral changes following low-dose in utero bisphenol A exposure. Proc Natl Acad Sci U S A. 2013 Jun;110(24):9956-61.

38 Szulwach KE, Li X, Li Y, Song C-X, Wu H, Dai Q, et al. 5-hmC-mediated epigenetic dynamics during postnatal neurodevelopment and aging. Nature Neuroscience. 2011 Dec;14(12):1607-16.

39 Schwarz JM, Nugent BM, McCarthy MM. Developmental and hormone-induced epigenetic changes to estrogen and progesterone receptor genes in brain are dynamic across the life span. Endocrinology. 2010 Oct;151(10):4871-81.

40 Musatov S, Chen W, Pfaff DW, Mobbs CV, Yang X-J, Clegg DJ, et al. Silencing of estrogen receptor alpha in the ventromedial nucleus of hypothalamus leads to metabolic syndrome. Proc Natl Acad Sci USA. 2007 Feb;104(7):2501-6.

41 Correa SM, Newstrom DW, Warne JP, Flandin P, Cheung CC, Lin-Moore AT, et al. An estrogenresponsive module in the ventromedial hypothalamus selectively drives sex-specific activity in females. Cell Rep. 2015 Jan;10(1):62-74. 
42 Cone RD, Cowley MA, Butler AA, Fan W, Marks DL, Low MJ. The arcuate nucleus as a conduit for diverse signals relevant to energy homeostasis. International Journal of Obesity. 2001 Dec;25(5):S63-7.

43 Scott CJ, Tilbrook AJ, Simmons DM, Rawson JA, Chu S, Fuller PJ, et al. The distribution of cells containing estrogen receptor-alpha (ERalpha) and ERbeta messenger ribonucleic acid in the preoptic area and hypothalamus of the sheep: comparison of males and females. Endocrinology. 2000 Aug;141(8):2951-62.

44 Kruijver FPM, Balesar R, Espila AM, Unmehopa UA, Swaab DF. Estrogen receptor- $\alpha$ distribution in the human hypothalamus in relation to sex and endocrine status. Journal of Comparative Neurology. 2002;454(2):115-39.

45 Voigt C, Ball GF, Balthazart J. Sex differences in the expression of sex steroid receptor mRNA in the quail brain. J Neuroendocrinol. 2009 Dec;21(12):1045-62.

46 Hiraki T, Takeuchi A, Tsumaki T, Zempo B, Kanda S, Oka Y, et al. Female-specific target sites for both oestrogen and androgen in the teleost brain. Proceedings of the Royal Society B: Biological Sciences. 2012 Dec;279(1749):5014-23.

47 Pradhan A, Olsson P-E. Zebrafish sexual behavior: role of sex steroid hormones and prostaglandins. Behav Brain Funct. 2015 Aug;11(1):23.

48 Kühnemann S, Brown TJ, Hochberg RB, MacLusky NJ. Sexual differentiation of estrogen receptor concentrations in the rat brain: effects of neonatal testosterone exposure. Brain Research. 1995 Sep;691(1):229-34.

49 Bakker J, Pool CW, Sonnemans M, Van Leeuwen FW, Slob AK. Quantitative estimation of estrogen and androgen receptor-immunoreactive cells in the forebrain of neonatally estrogen-deprived male rats. Neuroscience. 1997 Feb;77(3):911-9.

50 Kudwa AE, Harada N, Honda S-I, Rissman EF. Effects of organisational oestradiol on adult immunoreactive oestrogen receptors $(\alpha$ and $\beta$ ) in the male mouse brain. Journal of Neuroendocrinology. 2007;19(10):767-72.

51 Mellén M, Ayata $P$, Heintz N. 5-hydroxymethylcytosine accumulation in postmitotic neurons results in functional demethylation of expressed genes. PNAS. 2017 Sep;114(37):E7812-21.

52 Yu M, Hon GC, Szulwach KE, Song C-X, Jin P, Ren B, et al. Tet-assisted bisulfite sequencing of 5hydroxymethylcytosine. Nat Protoc. 2012 Dec;7(12):2159-70.

53 Kurian JR, Olesen KM, Auger AP. Sex differences in epigenetic regulation of the estrogen receptoralpha promoter within the developing preoptic area. Endocrinology. 2010 May;151(5):2297-305.

54 Liu XS, Wu H, Ji X, Stelzer Y, Wu X, Czauderna S, et al. Editing DNA methylation in the mammalian genome. Cell. 2016 Sep;167(1):233-247.e17.

55 Liu XS, Wu H, Krzisch M, Wu X, Graef J, Muffat J, et al. Rescue of fragile X syndrome neurons by DNA methylation editing of the FMR1 gene. Cell. 2018 22;172(5):979-992.e6. 
56 Kos M, O'Brien S, Flouriot G, Gannon F. Tissue-specific expression of multiple mRNA variants of the mouse estrogen receptor alpha gene. FEBS Lett. 2000 Jul;477(1-2):15-20.

57 Kulis M, Heath S, Bibikova M, Queirós AC, Navarro A, Clot G, et al. Epigenomic analysis detects widespread gene-body DNA hypomethylation in chronic lymphocytic leukemia. Nat Genet. 2012 Nov;44(11):1236-42.

58 Varley KE, Gertz J, Bowling KM, Parker SL, Reddy TE, Pauli-Behn F, et al. Dynamic DNA methylation across diverse human cell lines and tissues. Genome Res. 2013 Mar;23(3):555-67.

59 Fürst RW, Kliem H, Meyer HHD, Ulbrich SE. A differentially methylated single CpG-site is correlated with estrogen receptor alpha transcription. J Steroid Biochem Mol Biol. 2012 May;130(1-2):96-104.

60 Weaver ICG, Cervoni N, Champagne FA, D'Alessio AC, Sharma S, Seckl JR, et al. Epigenetic programming by maternal behavior. Nature Neuroscience. 2004 Aug;7(8):847-54.

61 Kriaucionis S, Heintz N. The nuclear DNA base, 5-hydroxymethylcytosine is present in brain and enriched in Purkinje neurons. Science. 2009 May;324(5929):929-30.

62 Lauber AH, Mobbs CV, Muramatsu M, Pfaff DW. Estrogen receptor messenger RNA expression in rat hypothalamus as a function of genetic sex and estrogen dose*. Endocrinology. 1991 Dec;129(6):3180-6.

63 El-Maarri O, Walier M, Behne F, van Üüm J, Singer H, Diaz-Lacava A, et al. Methylation at global LINE-1 repeats in human blood are affected by gender but not by age or natural hormone cycles. PLoS One. 2011 Jan;6(1). DOI: 10.1371/journal.pone.0016252

64 Wang L, Ozark PA, Smith ER, Zhao Z, Marshall SA, Rendleman EJ, et al. TET2 coactivates gene expression through demethylation of enhancers. Sci Adv. 2018;4(11):eaau6986. 


\section{Figure Legends}

Fig. 1. A sex difference in ERa labeling in the ARC emerges at weaning. $(a, c)$ Males and females have similar ERa labeling one day after birth (P1). (b,d) Females have more ERa labeling than males at P25. Mean + standard error of the mean and individual data points are depicted. Scale bar $=50$ $\mu \mathrm{m} .3 \mathrm{~V}=$ third ventricle

Fig. 2. The sex difference in ERa labeling in the ARC is programmed by neonatal testosterone and is eliminated by a neonatal inhibition of DNA methyltransferases. (A) Mean ERa labeling at P25 in control males and females and in females treated with testosterone $(T)$ at birth. Animals also received ICV injections of vehicle (left) or zebularine (right) during the first two days of life. Bars marked by different letters are significantly different $(P<0.01)$. (B) Photomicrographs showing ERa labeling in the six groups. Scale bar $=50 \mu \mathrm{m}$.

Fig. 3. Zebularine treatment does not alter cell death on P1 or total cell counts in the ARC at weaning. (A) Number of cells in the ARC that were positive for activated caspase-3 (AC3) in males and females treated with vehicle or zebularine on P0 and P1. Animals were sacrificed $3 \mathrm{~h}$ after the last injection. (B) Total neuron number in the ARC of P25 females that were treated with vehicle or zebularine neonatally. Mean + standard error of the mean and individual data points are depicted.

\section{Fig. 4. Sex differences in total DNA methylation of the Esr1 Exon A promoter emerge at P25, and in} opposite directions in the ARC and VMHvl. (A) Average total percent methylation across all 11 CpG sites in Exon A of the Esr1 promoter in the ARC (left) and VMHvl (right) at P1 and P25 in male and female mice. Overall methylation increased markedly in both brain regions from P1 to $P 25$. There were no significant sex differences at P1. At P25, total methylation was higher in females than in males in the ARC and higher in males than in females in the VMHvl. Mean + standard error of the mean and individual data points are depicted. (B) The sex difference in total methylation for individual CpG sites of Exon A at P25. Female minus male percent difference is plotted, e.g., [(Female \% methylation - Male \% methylation) / Male \% methylation $x 100$ ), so that a difference of +20 means $20 \%$ more methylation in females at that site. In the ARC (left), most CpG sites had greater total methylation in females, although this was significant only for CpG 10 in posthoc tests (asterisk). In the VMHvl, most CpG sites had 
greater total methylation in males, and this was significant for $\mathrm{CpG}$ 6, 9, 10 and

11 in posthoc tests (asterisks).

Fig. 5. Neonatal downregulation of Tets abolishes sex differences in ERa labeling in the ARC, but not VMHvl. Mean ERa labeling at P25 in control males and females and in males with neonatal Tet2 and Tet3 knock-down in the (A) ARC and (B) VMHvl. Bars marked by different letters are significantly different $(P<0.05)$. (C) Photomicrographs showing ERa labeling in the $A R C$ and VMHvl in the three groups. Scale bar $=50 \mu \mathrm{m}$.

Suppl. Fig. 1. There were no sex differences in average total methylation in Exon C of Esr1, but there was a subtle effect of age. Average total methylation across $5 \mathrm{CpG}$ sites in Exon C of the Esr1 promoter in the ARC (left) and VMHvl (right) at P1 and P25 in male and female mice. There were no significant sex differences at P1 or P25 in the ARC (left) or VMHvl (right).

Suppl. Fig. 2. Zebularine increases total methylation in Exon A of Esr1 in the ARC. Average total methylation across $11 \mathrm{CpG}$ sites in Exon A of the Esr1 promoter in the ARC was calculated for each animal. Zebularine-treated males and females had greater total methylation than vehicletreated animals.

Suppl. Fig. 3. Confirmation of Tet2 and Tet2 knock-down 48 hours after siRNA intracerebroventricular injection. There was a modest, but significant reduction in Tet2 (top) and Tet3 (bottom) expression in tissue punches from the anterior and posterior hypothalamus (main effect of siRNA treatment, with no interaction). 
bioRxiv preprint doi: https://doi.org/10.1101/2021.03.25.437030; this version posted March 26, 2021. The copyright holder for this preprint (which was not certified by peer review) is the author/funder. All rights reserved. No reuse allowed without permission.

\section{Figure 1}

a

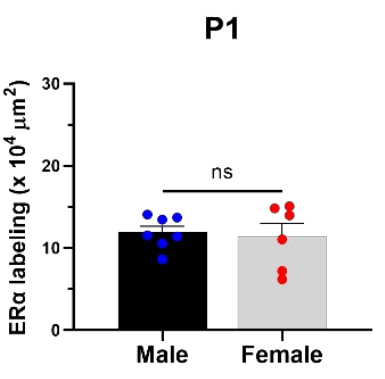

C

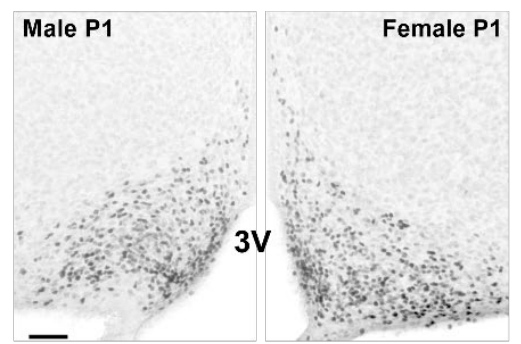

b

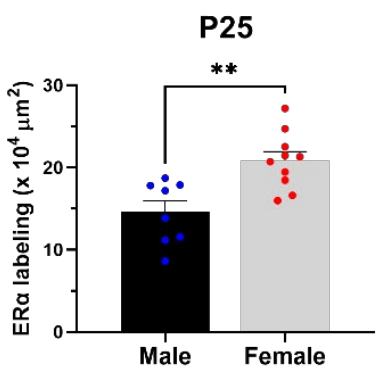

d

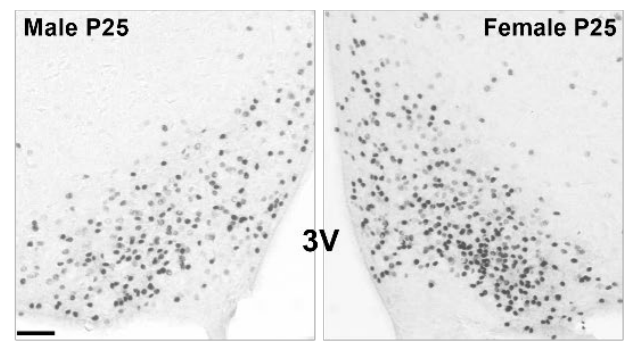


Figure 2
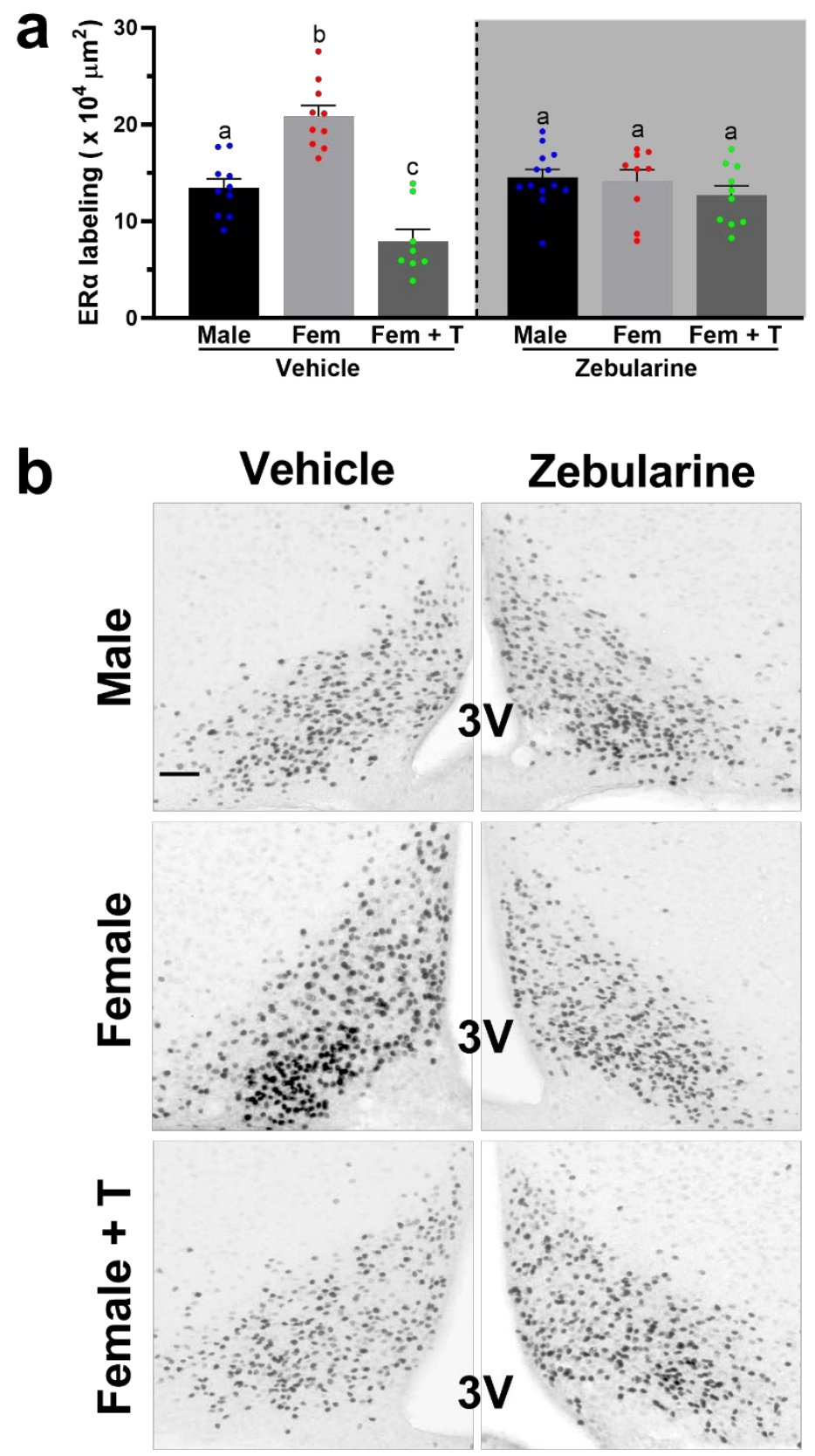
Figure 3

a

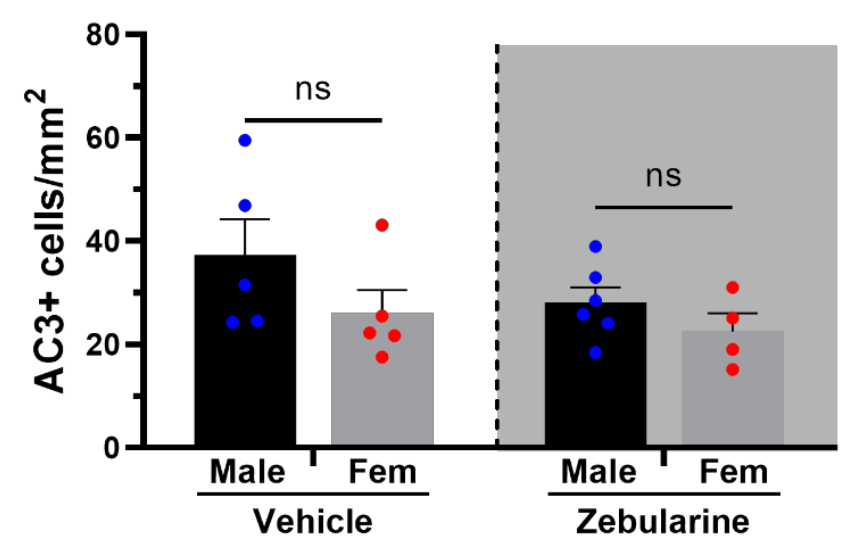

b P25

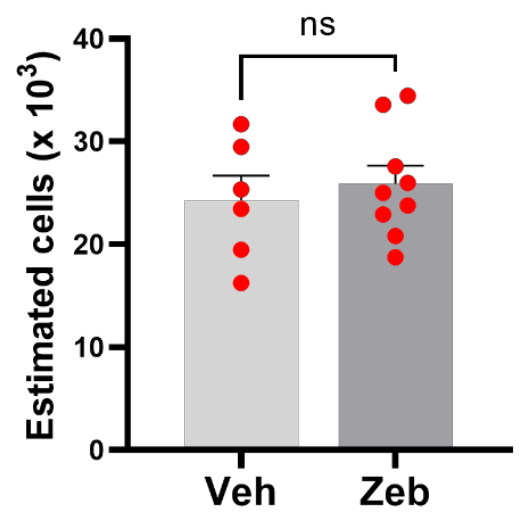


Figure 4

a

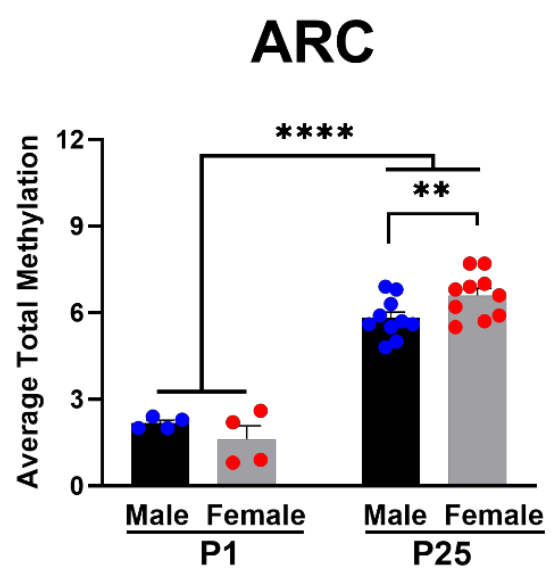

b

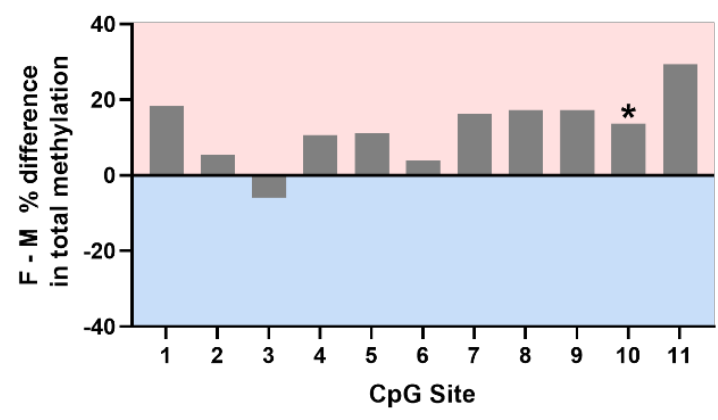

VMHvl
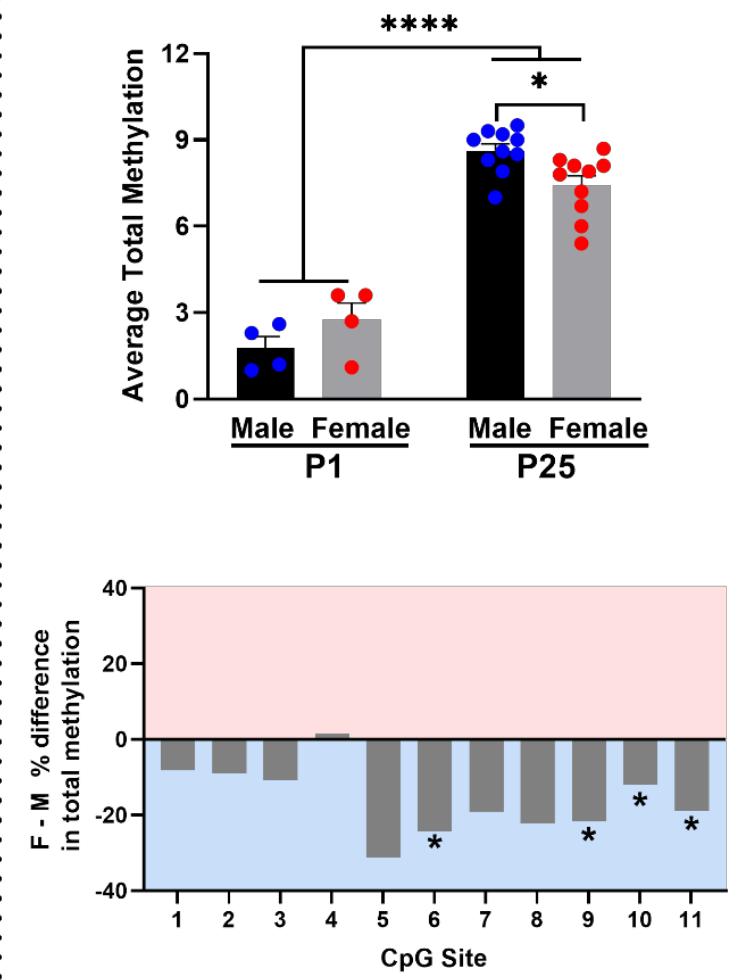


\section{Figure 5}

a

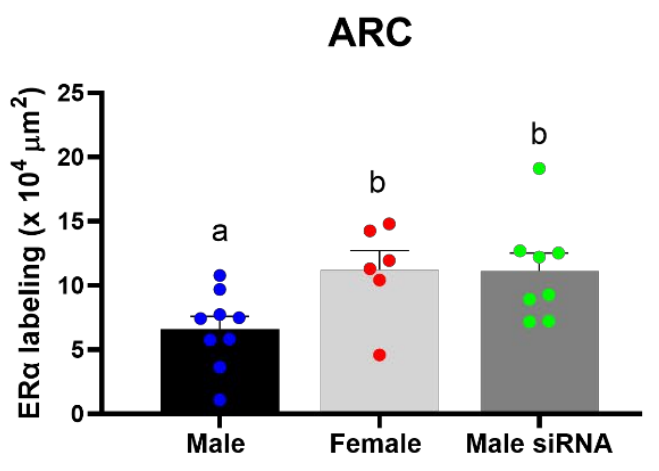

b

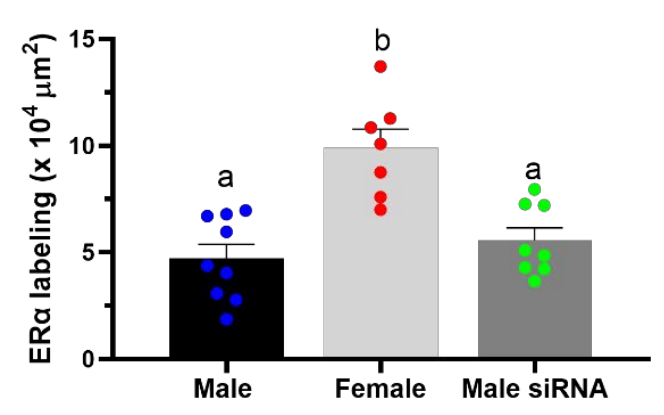

C

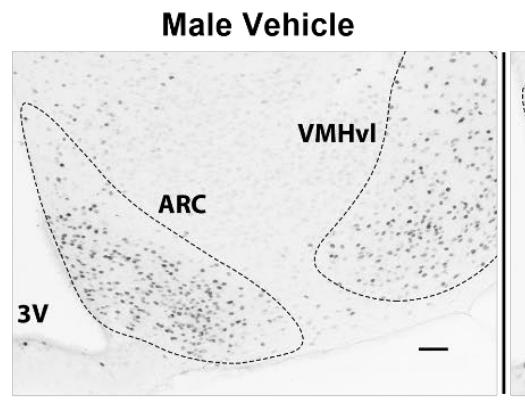

Female Vehicle

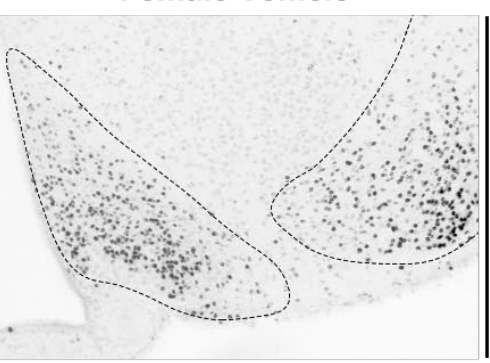

Male SiRNA

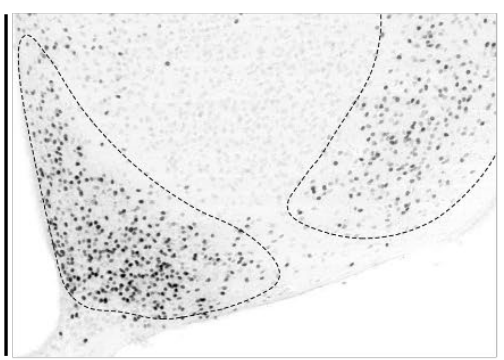

